\title{
The ISM of Low Metallicity Galaxies: The Herschel view
}

\section{Suzanne C. Madden ${ }^{1}$, Aurélie Rémy ${ }^{1}$, Frédéric Galliano ${ }^{1}$, Maud Galametz $^{2}$, George Bendo ${ }^{3}$, Diane Cormier ${ }^{1}$, Vianney Lebouteiller ${ }^{1}$, Sacha Hony ${ }^{1}$ and Herschel SAG 2 consortium}

\author{
${ }^{1}$ CEA Saclay, DSM, AIM, Service d'Astrophysique, \\ Gif-sur-Yvette 91911, France, email: suzanne.madden@cea.fr \\ ${ }^{2}$ Institute of Astronomy, University of Cambridge, Madingly Rd., Cambridge, UK \\ ${ }^{3}$ Alma Regional Center, University of Manchester, Oxford Rd., Machester, UK
}

\begin{abstract}
The wide variety of low metallicity galaxies of the local universe serve as convenient laboratories to study the evolution of gas and dust and conditions for star formation in environments which may resemble those of the early universe. The Herschel Dwarf Galaxy Survey is studying the far infrared (FIR) and submillimeter (submm) properties of the gas and dust in galaxies with metallicity values as low as $1 / 45$ that of solar. With complementary Spitzer, Laboca/APEX, Scuba/JCMT data, the dust spectral energy distributions are well constrained now, providing more accurate dust masses. We find a steep drop in dust-to-gas mass ratio $(\mathrm{D} / \mathrm{G})$ when the metallicity is below $12+\log (\mathrm{O} / \mathrm{H}) \sim 8$. A submillimeter excess can be found in some low metallicity galaxies, which, when present, becomes apparent at wavelengths at or longer than $500 \mu \mathrm{m}$. While $\mathrm{CO}$ is difficult to observe in low metallicity gas, the FIR fine structure lines, on the other hand, are very luminous and highlight a potentially important reservoir of CO-free molecular gas, better traced by the $158 \mu \mathrm{m}$ [CII] line.
\end{abstract}

Keywords. galaxies: dwarf — galaxies: ISM — ISM: molecules

\section{Introduction}

Dwarf galaxies, the most numerous galaxies in our local universe, are thought to be important actors in the formation and evolution of the universe. Our knowledge of the gas and dust properties and the intimate interplay between the star formation and interstellar medium (ISM) in dwarf galaxies is rapidly growing with recent mid-infrared (MIR), far-infrared (FIR) and submillimeter (submm) observations, most recently with the Herschel Space Observatory. Since the first IR space mission, IRAS, it was already known that the dust properties of the dwarf galaxies were different from their more metal-rich counterparts, with lower $\mathrm{L}_{I R}$ compared to $\mathrm{H} \alpha$ than spirals. $\mathrm{L}_{12 \mu \mathrm{m}} / \mathrm{L}_{25 \mu \mathrm{m}}$ values were lower than spirals, while the higher $\mathrm{L}_{60 \mu \mathrm{m}} / \mathrm{L}_{100 \mu \mathrm{m}}$ highlighted the presence of warmer dust dominating the significant MIR wavelength domain (e.g. Hunter et al. 1989; Melisse \& Israel 1994). The Infrared Space Observatory (ISO) and the Spitzer Space Observatory (Spitzer) brought to light the rich MIR spectroscopic signatures of the low metallicity star forming galaxies highlighting their proliferation for high excitation fine structure ionic lines on galaxy-wide scales. Also in the MIR, the scarcity of polycyclic aromatic hydrocarbons (PAHs) - the seemingly ubiquitous signature of more metal-rich star forming galaxies peaking in photodissociation regions (PDRs) around star-forming regions, was attributed to indirect effects of the low dust abundance: the intrinsic hard radiation field, destruction effects of shocks or the effect of delayed-injection of carbon by the evolved stars (e.g. Madden et al. 2006; Wu et al. 2006; O'Halloran et al. 2008; Galliano et al. 2008). One issue that has been plaguing confident determination of dust masses in dwarf 
galaxies has been an enigmatic submm excess flux observed beyond that explained by the typical dust SED models, becoming apparent sometimes at low metallicity, beginning near or beyond $500 \mu \mathrm{m}$. Before the Herschel Space Observatory (Herschel), SCUBA on the JCMT and Laboca on APEX uncovered this excess emission in dwarf galaxies at $850 / 870 \mu \mathrm{m}$ which, if interpreted as cold dust, would comprise an excessive amount of dust in low metallicity galaxies compared to their measured gas reservoirs and their low metallicities (e.g. Galliano et al. 2003; Galliano et al. 2005; Zhu et al. 2009; Galametz et al. 2011). Likewise, quantifying the total gas mass is a perplexing issue, since $\mathrm{CO}(1-0)$, the observable we take for granted to trace the molecular hydrogen, is difficult to detect, if present at all, in dwarf galaxies (see Schruba et al. 2012 and references within). On the other hand, luminous $158 \mu \mathrm{m}$ [CII] emission has been noted in dwarf galaxies originating with the Kuiper Airborne Observatory (KAO) and later with the FIR spectrometer, LWS, on ISO (Stacey et al. 1991; Poglitsch et al. 1995; Madden et al. 1997; Madden et al. 2000; Bergvall et al. 2000; Hunter et al. 2001; Vermeij et al. 2002; Brauher et al. 2008; Israel et al. 2011). The [CII] line is thought to mostly originate from photodissociated regions (PDRs), the UV-illuminated surfaces of molecular clouds. The UV photons dissociate CO molecules and ionise these atoms in the lower dust abundance PDR shell, leaving a relatively small $\mathrm{CO}$ core. Since $\mathrm{H}_{2}$ can remain self-shielded in this region, this 'missing' reservoir of $\mathrm{H}_{2}$, not traced by $\mathrm{CO}$, can be substantial in low metallicity conditions (Wolfire et al. 2010). Using [CII] to uncover this CO-free gas was first demonstrated in the Local Group, low metallicity galaxy, IC10 (Madden et al. 1997), where as much as 100 times more molecular gas was suggested to be present, compared to that traced by $\mathrm{CO}$ alone. These uncertainties in the gas and dust properties render the ISM of low metallicity galaxies and the conditions for star formation very elusive.

One way to pin down the CO-dark gas mass in low metallicity galaxies, has been to determine the dust mass from the observed SED, quantify the gas mass from $\mathrm{HI}$ and $\mathrm{CO}$ observations and presume a dust-to-gas mass ratio $(\mathrm{D} / \mathrm{G})$ linearly scaled to the metallicity of the galaxy. The difference between the expected gas mass and that observed is the missing gas mass. This technique has uncovered a CO-dark reservoir of molecular gas in galaxies and has become a popular approach with Herschel and Planck data. This often relies on assumptions of the FIR/submm dust model, the conversion between $\mathrm{CO}$ and $\mathrm{H}_{2}$ gas mass and presumed local or global $\mathrm{D} / \mathrm{G}$ values.

Herschel has opened up the the FIR and submm wavelength window to high sensitivity, high spatial resolution (10" to 38") observations of gas and dust tracers in the 50 to 500 $\mu \mathrm{m}$ window, giving access to the numerous low luminosity dwarf galaxies. The Dwarf Galaxy Survey (DGS; P.I. Madden) is a Herschel key program targeting 48 local universe dwarf galaxies with a wide range of star formation properties and metallicity values, as low as $1 / 50 \mathrm{Z} \odot$ (Fig. 1) and as nearby as the Magellanic Clouds, to study the multiphase components of the stars, gas and dust under low metallicity environments.

\section{SED models of dwarf galaxies}

PACS observations cover 3 photometric bands: 70, 100 and $160 \mu \mathrm{m}$ (FWHM $\sim 10$ "; Poglitsch et al. 2010) while SPIRE observes at 250, 350 and $500 \mu \mathrm{m}$ (FMHM=18" to 38"; Griffin et al. 2010). Comparison of the Spitzer $24 \mu \mathrm{m}$ images (Bendo et al. 2012) with the PACS $70 \mu \mathrm{m}$ and SPIRE $250 \mu \mathrm{m}$ (Fig. 1) highlights the extent of the cooler dust traced by the $250 \mu \mathrm{m}$ emission in contrast to the warmer dust emitting at $24 \mu \mathrm{m}$, originating from the young star clusters at the source of the compact HII regions. Observations at Spitzer and Herschel wavelengths together provide well-sampled SEDs for in depth studies of the dust properties in dwarf galaxies. 

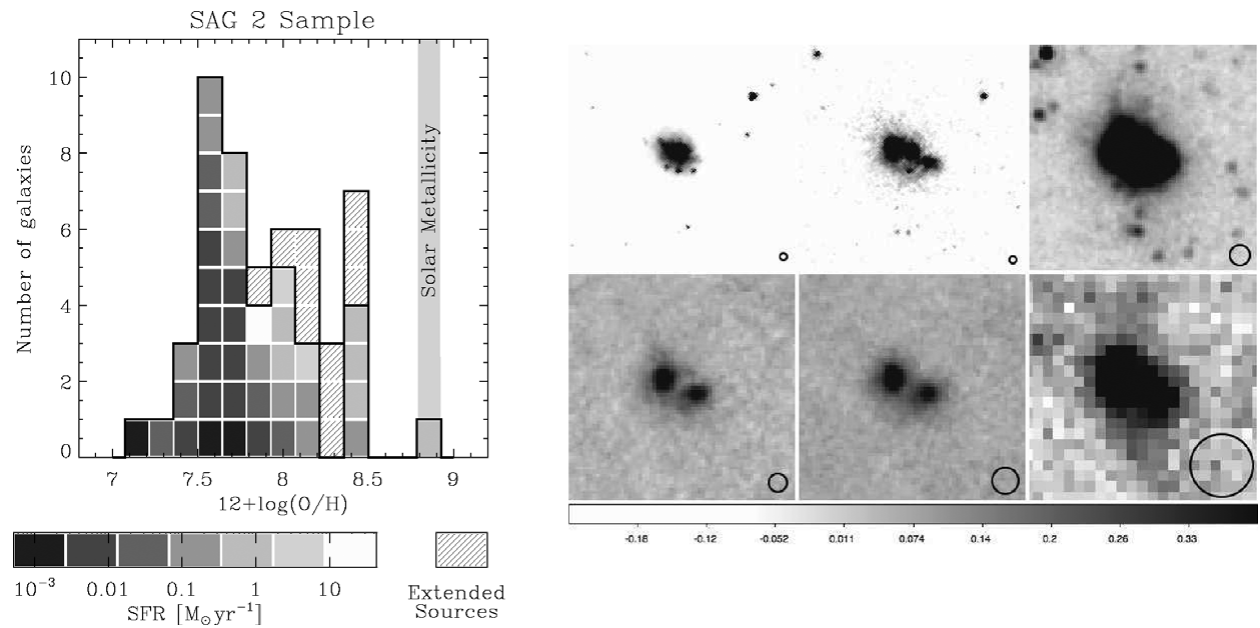

Figure 1. (left) Metallicity and star formation properties of the Herschel Dwarf Galaxy Survey. (right) Spitzer and Herschel images of the dwarf galaxy, NGC 1705. Images from left to right and top to bottom are: $3.6 \mu \mathrm{m}$ Spitzer/IRAC, $8 \mu \mathrm{m}$ Spitzer/IRAC, $24 \mu \mathrm{m}$ Spitzer/MIPS, $70 \mu \mathrm{m}$ Herschel/PACS, $100 \mu \mathrm{m}$ Herschel/PACS, $250 \mu \mathrm{m}$ Herschel/SPIRE. Image sizes are 130"x130" and beam sizes are indicated in lower right corner of images.

$\beta$ and T effects and metallicity. A Spitzer and Herschel color-color diagram of the full sample (Fig. 2) provides a first view of FIR-submm behavior of the DGS sample (Rémy et al. in preparation). The effects of varying emissivity indices $(\beta)$ and average dust temperatures $(\mathrm{T})$, presuming a single modified black-body to explain the FIR-submm emission, can be seen. For comparison the KINGFISH galaxies (Dale et al. 2012) are also included. The effect of the overall higher metallicity of most of the KINGFISH sample places these galaxies between $\beta=1.5$ and 2.0. The most active star-forming dwarf galaxies, particularly the blue compact dwarfs, peak at wavelengths shortward of $70 \mu \mathrm{m}$ and some between 35 and $60 \mu \mathrm{m}$, much shorter wavelengths compared to the more metalrich starburst galaxies. The difference in the shape of the SEDs of dwarf galaxies can be noted in the Herschel color-color diagram: as a consequence of their overall hotter dust peaking at MIR wavelengths (higher PACS70/PACS160 values for the lower metallicity bins), their Rayleigh-Jeans slope drops off at FIR and submm wavelengths. Consequently, some of the lowest metallicity galaxies are often not detected at all SPIRE wavelengths, particularly at $500 \mu \mathrm{m}$. A prominent example of this effect is illustrated in one of the lowest metallicity galaxies of our sample, SBS0335-052E $(12+10 g(\mathrm{O} / \mathrm{H})=7.29$; Fig. 3). The integrated SED has a flatter MIR-FIR emission peaking between 20 and $30 \mu \mathrm{m}$ (Houck et al. 2004; Galliano et al. 2008. The very dense super star clusters dominate the observed warm overall dust emission, with the observed SED leaving little evidence for prominent cold, submm-emitting dust.

For those dwarf galaxies detected at both PACS and SPIRE wavelengths, modified black-body fits (omitting $70 \mu \mathrm{m}$ in the fits) give a wide range of $\beta$ and $\mathrm{T}$ solutions with a mean $\beta$ of 1.6 and mean $\mathrm{T}$ of $32 \mathrm{~K}$ (Fig. 4). Since the most metal-poor galaxies of the DGS sample do not have $500 \mu \mathrm{m}$ detections, this distribution of modeled $\beta$ and $\mathrm{T}$ is primarily representing galaxies with metallicity values greater than $12+\log (\mathrm{O} / \mathrm{H}) \sim$ 8.0 - 8.2. For comparison, the KINGFISH galaxies show a similar mean $\beta$ but the $\mathrm{T}$ distributions are cooler, peaking between $\sim 20$ and $25 \mathrm{~K}$ (Dale et al. 2012). Galaxies requiring an exceptionally low $\beta$ solution, may be indicative of the presence of a submm excess which could start to be detectable at wavelengths as low as $500 \mu \mathrm{m}$. 


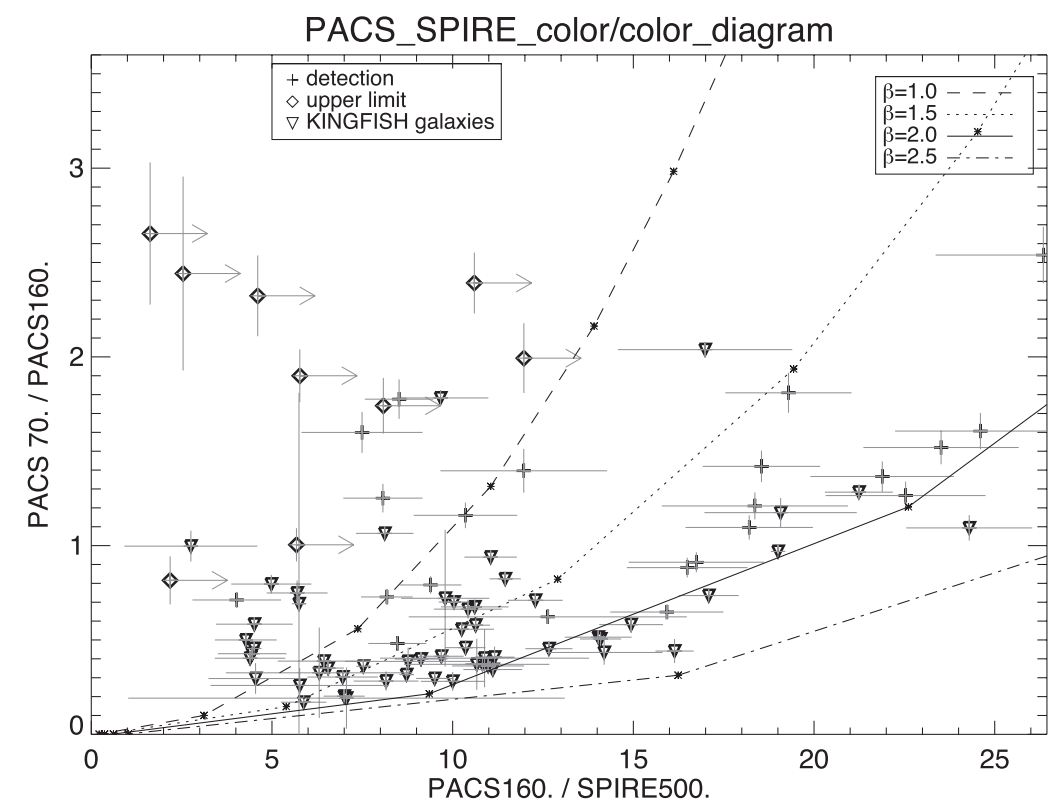

Figure 2. Herschel color-color diagram of DGS galaxies detected in at least all PACS bands: PACS70/160 and PACS160/SPIRE500. For comparison, KINGFISH galaxies (Dale et al. 2012) are also shown (downward triangles). Modeled modified black body fits with $\beta=1.0,1.5,2.0$, 2.5 are shown in curves, with temperature values noted as dots on the curves, increasing from $10 \mathrm{~K}$ in steps of $10 \mathrm{~K}$, starting from the lower left of the curves to the upper right.

Submm excess examples. Taking into account the optical to submm and radio wavelengths, full SED models (Galliano et al. 2008) can help to further interpret the Herschel color-color diagrams. For example, from the Herschel color-color diagram (Fig. 2), HS0052+2536 has an exceptionally low $\beta$ value $\sim 1$. Inspection of the fully modeled SEDs (Fig. 3) shows that a submm excess is indeed present. About $50 \%$ of the DGS galaxies detected at $500 \mu \mathrm{m}$ show a submm excess of $\sim 7 \%$ to $100 \%$ above the SED model (Rémy et al. in preparation). A relationship between metallicity and submm excess within the DGS sample is not yet obvious. This may be somewhat due to the requirement for $500 \mu \mathrm{m}$ detections which often omits the lowest metallicity galaxies. While this Herschel colorcolor diagram highlights potential galaxies with submm excess, galaxies for which the excess begins beyond $500 \mu \mathrm{m}$ will be missed without observations at longer wavelengths. For example, NGC 1569 and He2-10, show $\beta \sim 2$ in the Herschel color-color diagram but do indeed show a submm excess when including their ground-based $850 \mu \mathrm{m}$ observations (Fig. 3; see also Galliano et al. 2003; Galliano et al. 2005; Galametz et al. 2011).

$D / G$ and possible origins of the submm excess. Understanding how the $D / G$ varies as a function of metallicity is important to have an accurate picture of the gas and dust life cycle in galaxies. How are the heavy metals incorporated into dust and how do metallicity or other local or global parameters control this process? Understanding the behavior of the $\mathrm{D} / \mathrm{G}$ is also important since many studies determining the dust mass in galaxies then quantify the gas reservoir in galaxies by assuming a D/G. Numerous studies have noted a proportionality of $\mathrm{D} / \mathrm{G}$ with metallicity, but results can vary depending on the long wavelength data used. Engelbracht et al. (2008) noted a decrease in $\mathrm{D} / \mathrm{G}$ from the more metal rich galaxies to the moderately metal-poor galaxies, until $12+\log (\mathrm{O} / \mathrm{H}) \sim$ 8 , beyond which the $\mathrm{D} / \mathrm{G}$ appears to be constant. Adding $850 \mu \mathrm{m}$ observations beyond Spitzer wavelengths, however, can increase dust masses (Galametz et al. 2011). The low 

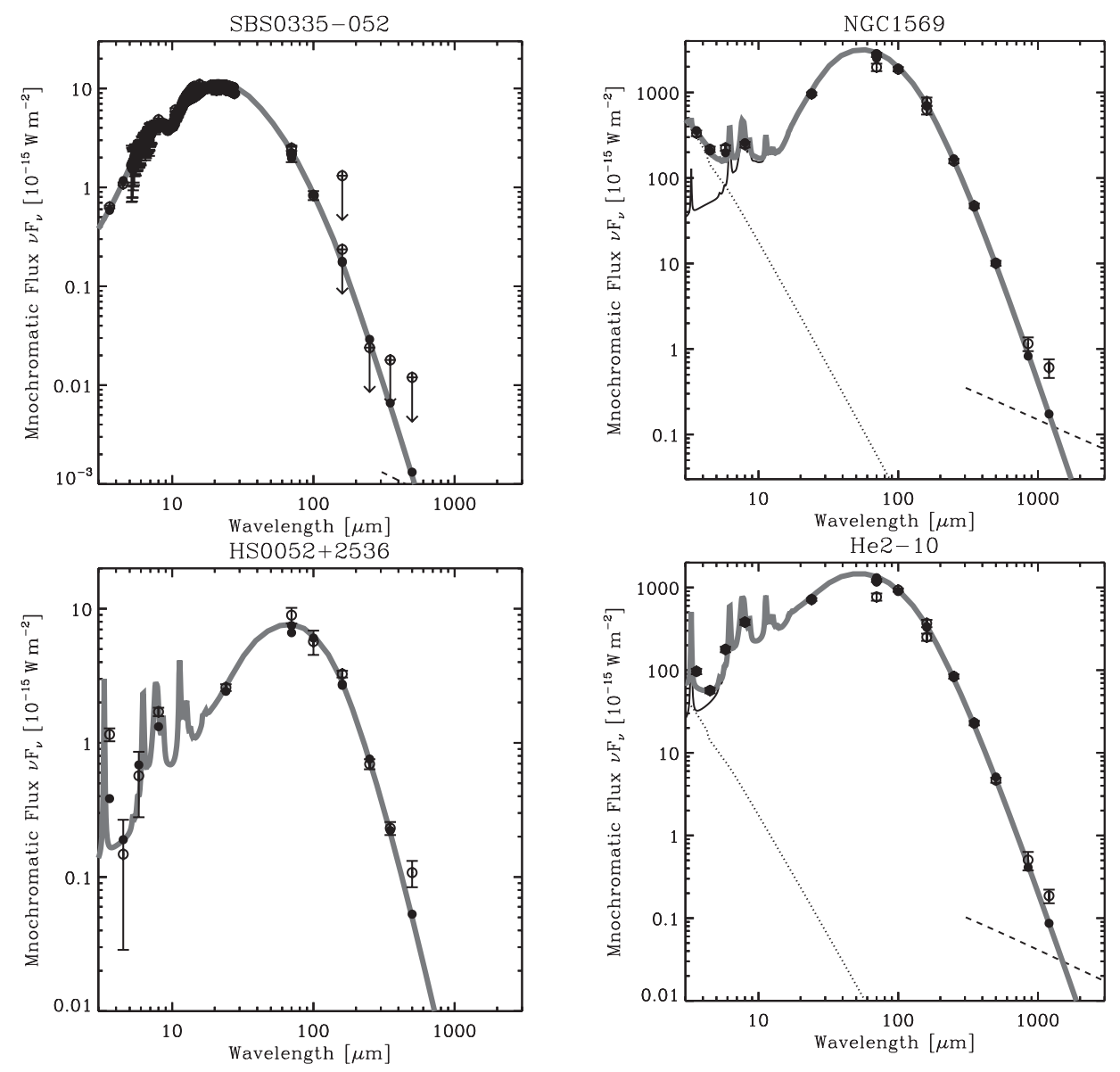

Figure 3. SEDs of dwarf galaxies. SBS0335-052 has on average very hot dust, with the SED peaking between 20 and $30 \mu \mathrm{m}$ (Galliano et al. 2008; Sauvage et al. in preparation]). HS0052+2536 shows a very low $\beta$ in the Herschel color-color diagram (Fig. 2) and does indeed show a $500 \mu \mathrm{m}$ excess. NGC 1569 and He2-10 both fall near $\beta \sim 2$ in the color-color diagram, but their full SED model unveils an excess beyond $500 \mu \mathrm{m}$. Spitzer and Herschel data are used to constrain the SED models. Herschel observations are used for the model constraints in the cases of data redundancy $(70$ and $160 \mu \mathrm{m})$. Stellar contribution is shown as dotted lines, the dashed lines are the free-free continuum, extrapolated from observed radio observations when available and the solid grey curve is the total modeled SED. The open squares are the observations while the black points show the predicted model fluxes, often overlapping completely with the opens squares.

metallicity end of the $\mathrm{D} / \mathrm{G}$ relationship has been particularly ambiguous, since dust mass estimates of dwarf galaxies are sometimes hampered by a submm excess.

For the lowest metallicity galaxies $(12+\log (\mathrm{O} / \mathrm{H})<8.0)$ where the overall SED peaks at short wavelengths and where we have mostly only upper limits in the submm, we find low upper limits to dust masses and low D/G compared to the expected value. The upper limit dust mass from SBS0335-052, for example, is very low (Galliano et al. 2008) giving an unusually low total $\mathrm{D} / \mathrm{G}$, compared to that expected for its extremely low metallicity. The low D/G measured for the lowest metallicity galaxies may be telling us that perhaps metals are not necessarily incorporated into dust in the same way for 
the lowest metallicity galaxies as for the more metal rich galaxies. On the other hand, the total gas reservoir may be underestimated.
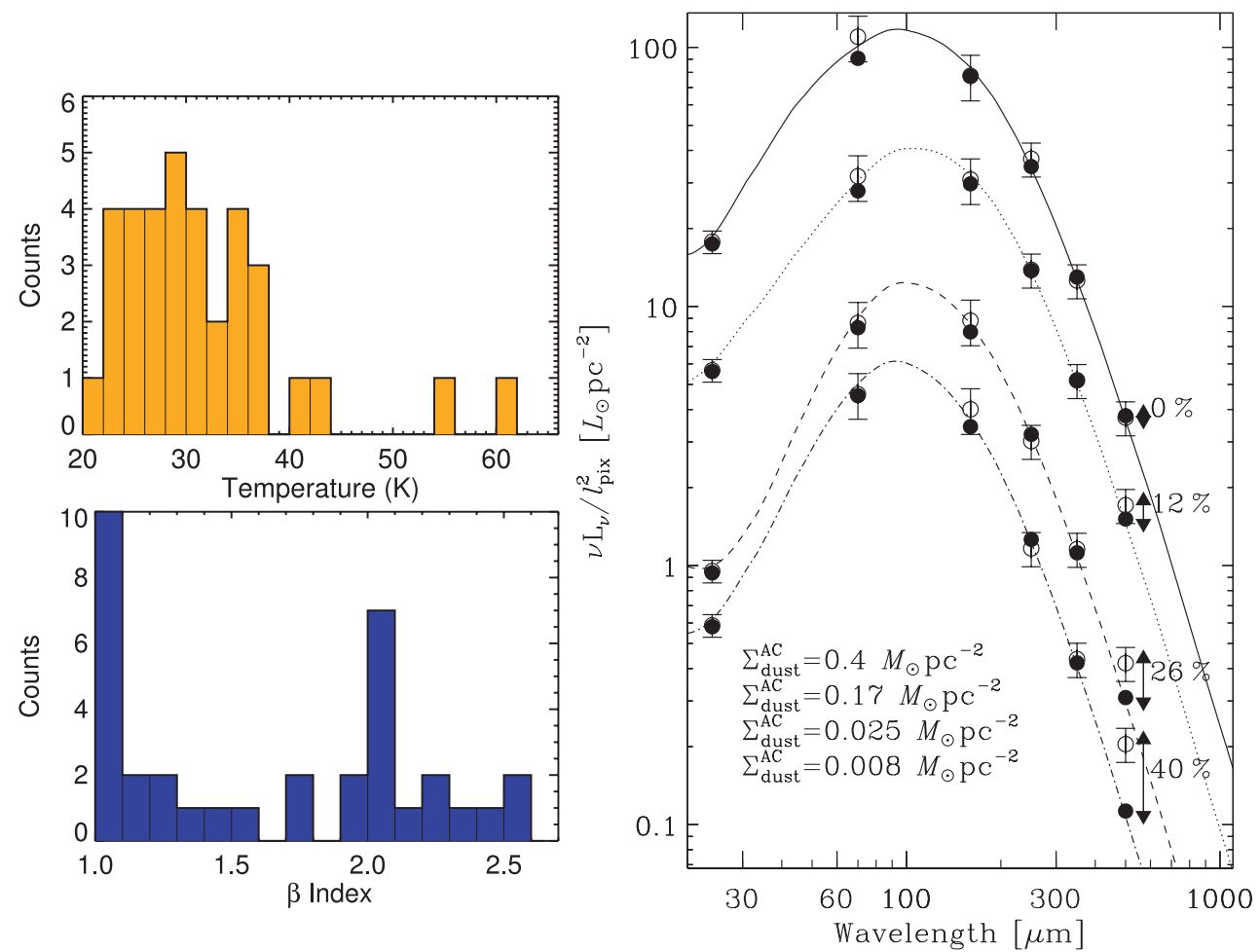

Figure 4. (left) $\mathrm{T}$ and $\beta$ distribution of the DGS sample with $500 \mu \mathrm{m}$ detections. (right) Selected SEDs in the LMC showing submm excess ranging up to $40 \%$ compared to that expected from the SED models (Galliano et al. 2011). SEDs are for a range of dust mass surface densities, ranging from the lowest to the highest values, corresponding to lower to higher profiles, respectively.

The submm excess has been noted in dwarf galaxies since the first SCUBA observations of dwarf galaxies at $850 \mu \mathrm{m}$. The origin of the submm excess remains uncertain. In the LMC, where Herschel brings $10 \mathrm{pc}$ resolution at $500 \mu \mathrm{m}$, Galliano et al. (2011) have highlighted locations of submm excess ranging from $15 \%$ to $40 \%$ compared to that expected from the SED models (Fig. 4). The excess seems to be anticorrelated with the dust mass surface density. Possible explanations for the submm excess include: 1) very cold dust component (e.g. Galliano et al. 2005; Galametz et al. 2011; 2) excessive free-free emission; 3) unusual dust emissivity properties (e.g. Lisenfeld et al. 2002; Mény et al. 2002; Galliano et al. 2011; Paradis et al. 2012); 4) anomalous spinning dust (e.g. Draine \& Lazarian 1998; Ysard et al. 2010; 5) magnetic nanoparticles (Draine \& Hensley). With more sensitive submm wavelength coverage, Herschel is confirming the flater submm slope in more dwarf galaxies. Problems exist with these explanations put forth for the origin of the excess. For example, invoking a very cold dust component to account for the submm excess can augment the dust mass excessively, resulting in a very high $D / G$ compared to that expected for the metallicity. But, are we correctly quantifying the total molecular gas mass in low metallicity environments? 

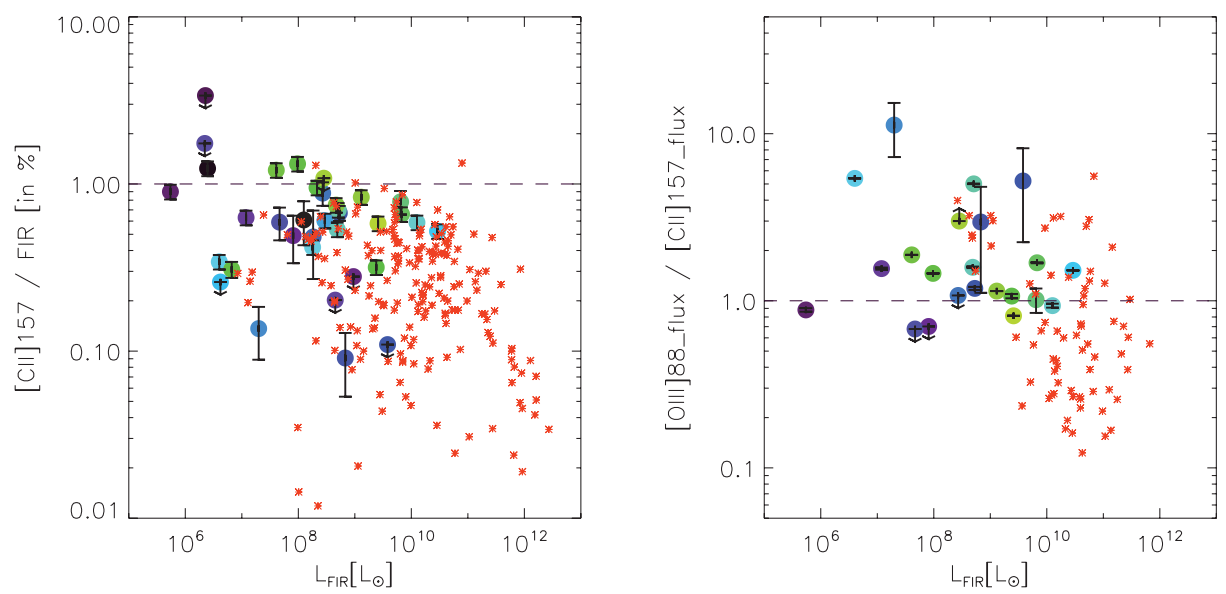

Figure 5. [CII]/FIR and [OIII]/FIR as a function of $\mathrm{L}_{[F I R]}$ for the DGS sample (large dots) compared to that of Brauher et al. 2008 ISO data (small squares) - mostly metal-rich galaxies.

\section{FIR fine structure lines in dwarf galaxies with Herschel}

The PACS spectrometer footprint consists of $5 \times 5$ pixels of 9.4 " each, with a total field of view of $47 " \times 47$ ". In only a few cases, were all of the brightest 7 FIR fine structure lines observed: $158 \mu \mathrm{m}$ [CII], 63 and $145 \mu \mathrm{m}$ [OI], $88 \mu \mathrm{m}$ [OIII], $57 \mu \mathrm{m}$ [NIII] and the 122 and $205 \mu \mathrm{m}$ [NII] lines. In all but a few of the faintest galaxies, at least the [CII] and [OIII] were observed. The spectral resolutions for the [CII], [OIII] and [OI] lines are: $1250(240 \mathrm{~km} \mathrm{~s}-1), \sim 2400(125 \mathrm{~km} \mathrm{~s}-1)$, and $\sim 3300(90 \mathrm{~km} \mathrm{~s}-1)$, respectively.

\section{The molecular gas in dwarf galaxies: [CII]}

While $\mathrm{CO}$ is the widely used means to access $\mathrm{M}_{H_{2}}$ in galaxies, it is by now a wellestablished fact that the Galactic X factor, which converts $\mathrm{CO}$ to $\mathrm{M}_{H_{2}}$, underestimates the mass of molecular gas in low metallicity galaxies. What is uncertain, however, is how to accurately correct up this factor as a function of metallicity to obtain the total gas reservoir. During the last 2 decades valiant effort has been invested in detecting and interpreting $\mathrm{CO}$ emission in low luminosity dwarf galaxies to quantify the molecular gas reservoir (e.g. Leroy et al. 2009 and references within). With the first detections of the $158 \mu \mathrm{m}[\mathrm{CII}]$ line in dwarf galaxies on the KAO, the surprisingly high [CII]/CO values highlighted the fact that $\mathrm{CO}$ could be missing a large reservoir of molecular gas (factors of 10 to 100) due to the lower dust abundance. The consequently deeper penetration of UV photons further photodissociate CO, reducing the CO cores - hence, the dearth of detected $\mathrm{CO}$. Due to the self-shielding of $\mathrm{H}_{2}$, the larger $\mathrm{C}^{+}$-emitting envelope can harbor $\mathrm{H}_{2}$ which is not accounted for via CO observations (e.g. Poglitsch et al. 1995; Madden et al. 1997; Wolfire et al. 2010). The observed [CII]/CO, thought to be a useful tracer of star formation in galaxies, can sometimes be a factor of a 2 to 5 (or more) times higher in dwarf galaxies than in metal-rich galaxies (e.g. Madden 2000; Stacey et al. 2010).

Dwarf galaxies generally emit a larger fraction of their FIR in the [CII] line, $\sim 0.5$ to $2 \%$, in contrast to the more metal-rich spirals and starbursts which usually show $[\mathrm{CII}] /$ FIR less than $\sim 0.5 \%$ (Fig. 5). If $[\mathrm{CII}]$ is the dominant coolant in galaxies and the photoelectric effect is responsible for the primary heating of the ISM, then $[\mathrm{CII}] / \mathrm{FIR}$ can be thought of as a proxy for the grain photoelectric heating efficiency (e.g. Rubin et al. 2009) - the fraction of the power being absorbed by the grains that goes into 
heating of the ISM. The high [CII]/FIR values observed in dwarf galaxies thus, imply high photoelectric heating efficiencies which may be attributed to properties of the lowmetallicity ISM, including lower dust abundance, clumpy ISM, etc. Traversing the galaxy, UV photons suffers less attenuation in the metal-poor ISM, and as a consequence of the longer photon mean-free path, the dust on galaxy-wide scales is subject to lower UV flux, effectively decreasing the overall FIR flux and increasing the observed [CII]/FIR. The $88 \mu \mathrm{m}$ [OIII] line is typically the brightest FIR line in dwarf galaxies - usually brighter than the [CII] line (Fig. 5), normally considered to be the brightest FIR line in galaxies. This suggests a substantial filling factor of ionized gas in dwarf galaxies and places an important constraint on quantifying the origin of the [CII] line, which also can be excited by electrons in the diffuse ionized gas, not only PDRs (Lebouteiller et al. 2012). The $[\mathrm{CII}]$ and [OIII] together are important calibrators of star formation activity and characterize the diffuse and molecular phases in galaxies. With ALMA, these lines are valuable accessible diagnostics of the ISM of high red-shift galaxies offering new insight on the evolution of the star formation and ISM throughout earlier epochs.

\section{References}

Bendo, G., Galliano, G., \& Madden, S. C. 2012, MNRAS submitted

Brauher, J. R. Dale, D. A., Helou, G. 2008 ApJS, 178, 280

Dale, D. A., Aniano, G., Engelbracht, C. W., Hinz, J. L., Krause, O. et al. 2012, ApJ, 745, 95 Draine, B. T. \& Lazarian, A. 1998, ApJ, 508, 157

Draine, B. T. \& Hensley, B. 2012, ApJ, 757, 103

Engelbracht, C. W., Rieke, G. H., Gordon, K. D., Smith, J.-D. T. et al. 2008, ApJ, 678, 804

Galametz, M., Madden, S. C., Galliano, F., Hony, S., \& Bendo, G. J. et al. 2011, A\& A, 532, 56

Galliano, F., Madden, S. C., Jones, A., \& Wilson, C., et al. 2003, A\&A, 407, 159

Galliano, F., Madden, S. C., Jones, A. P., Wilson, C. D., \& Bernard, J.-P. 2005, A\& A, 434, 867

Galliano, F., Dwek, E., \& Chanial, P. 2008, A $\& A$, 672, 214

Galliano, F., Hony, S., Bernard, J.-P., Bot, C., Madden, S. C. et al. 2011, A\& A, 536, 88

Griffin, M., Abergel, A., Abreu, A., Ade, P. A. R. et al. 2010, A\&3A, 518, 3

Houck, J. R., Charmandaris, V., Brandl, B. R., Weedman, D. et al. 2004, ApJS, 154, 211

Hunter, Deidre A., Gallagher, John S., \& III, Rice, Walter L., et al. 1989, A\&\&A, 336, 152

Israel, F. P., Maloney, P. R., Geis, N., Herrmann, F., Madden, S. C. et al. 1996, ApJ, 465, 738

Lebouteiller, V., Cormier, D., Madden, S. C., et al. 2012, A\&AA, submitted

Leroy, A. K., Walter, F., Bigiel, F., Usero, A., Weiss, A. et al. 2009, AJ, 137, 4670

Lisenfeld, U., Israel, F. P., Stil, J. M., \& Sievers, A.l 2002, A\& $A$, 382, 860

Madden, S. C., Poglitsch, A., Geis, N., Stacey, G. J., \& Townes, C. H. 1997, ApJ, 483, 200

Madden, S. C. 2000, NewAR, 44, 249

Madden, S. C., Galliano, F., Jones, A. P., \& Sauvage, M. 2006, A\& $A$, 446, 877

Melisse \& Israel 1994, $A \mathscr{E} A, 285,51$

Mény, C., Gromov, V., Boudet, N., \& Bernard, J.-Ph., et al. 2007, A\&̈A, 468, 171

O'Halloran, B., Madden, S. C., Abel, N. P. 2008 ApJ, 681, 1205

Paradis, D., Paladini, R., Noriega-Crespo, A., Mény, C. et al. 2012, A\&3A, 537, 113

Poglitsch, A., Krabbe, A., Madden, S. C., Nikola, T., Geis, N. et al. 1995, ApJ, 454, 293

Poglitsch, A., Waelkens, C., Geis, N., Feuchtgruber, H. et al. 2010, A\&\&A, 518, 1

Popescu, Cristina C., Tuffs, Richard J., Volk, Heinrich J. et al. 2002, ApJ, 567, 221

Rubin, D., Hony, S., Madden, S. C., \& Tielens, A. G. G. M. et al. 2009, A\&GA, 494, 647

Stacey, G. J., Hailey-Dunsheath, S., Ferkinhoff, C., Nikola, T. et al. 2010, ApJ, 724, 957

Wolfire, M. G., Hollenbach, D., \& McKee, C. F. 2010, ApJ, 716, 1191

Wu, Yanling, Charmandaris, V., Hao, Lei, Brandl, B. R., et al. 2006, ApJ, 639, 157

Ysard, N., Miville-Deschnes, M. A., \& Verstraete, L. 2010, A\&\&A 509,1

Zhu, M., Papadopoulos, P., \& Xilouris, et al. 2009, ApJ, 706, 941 